\title{
Delirium and functionality: The impact of delirium on the level of functioning
}

\section{Susanne Boettger William Breitbart** Josef Jenewein* Soenke Boettger}

* Department of Psychiatry and Psychotherapy, University Hospital Zürich, Zürich

** Department of Psychiatry and Behavioral Sciences, Memorial Sloan Kettering Cancer Center, New York

SWITZERLAND

USA

\begin{abstract}
Background and Objectives: Studies have shown showed that delirium affects the long-term functional status, however, the acute effect of delirium on functioning has been less documented. The purpose of this analysis was to examine the acute impact of delirium on the level of functioning.

Methods: All patients were recruited at the Memorial Sloan Kettering Cancer Center (MSKCC). The Memorial Delirium Assessment Scale (MDAS) and Karnofsky scale of Performance Status (KPS) were recorded at baseline (T1), 2-3 days (T2) and 4-7 days (T3). A secondary analysis in respect to sociodemographic and medical variables, aspects of delirium, and level of functioning was performed.

Results: Delirium severity at baseline did not affect the level of functioning, however, resolved delirium resulted in substantial functional recovery compared to persistent delirium at T2 (30.2 and 23.5) and T3 (35.1 and 26.2). Patients with persistent functional impairment were not different in age, pre-existing dementia or brain metastasis compared to those with functional recovery. However, brain cancer, terminal illness, hypoxia and multiple etiologies caused persistent functional decline. Although delirium severity was not different at baseline, delirium was more severe in the functionally-impaired at T2 and T3. Similarly, delirium resolution was inferior in these patients in contrast to the functionally-recovered at $\mathrm{T} 2(25 \%$ and $65.4 \%)$ and $\mathrm{T} 3(62.5 \%$ and $83.3 \%)$. On the contrary, the duration of delirium affected functional recovery; shorter delirium predicted faster functional recovery.

Conclusions: Delirium caused an acute functional decline and appropriate management of delirium with antipsychotics reversed this decline. On the contrary, shorter duration of delirium was associated with faster functional recovery. Brain cancer, terminal illness, hypoxia, and multiple etiologies were confounders for persistent delirium and functional impairment.
\end{abstract}




\section{Background}

Delirium is associated with poor functional outcome, increased morbidity and mortality, as well as prolonged hospitalization ${ }^{1}$, and is a common event in the course of hospitalization depending on the age of the patient and the severity of the illness ${ }^{2,3}$. In the general hospital setting, 14-24\% of older patients present with delirium on admission, and 6-56\% will develop delirium during the course of hospitalization ${ }^{4}$. In this setting, the occurrence of delirium is high and varies in medically ill patients between $15-30 \%$, in the hospitalized elderly between $10-40 \%$, in cancer patients between $57-85 \%$, and in terminal illness, the incidence of delirium can reach up to $85 \%$ 6-8.

Several studies have indicated that the incidence of delirium prolongs hospitalization and that in particular in older patients, delirium has an impact on the cognitive and functional status with or without dementia ${ }^{9-11}$. In addition, delirium has been shown to lead to an acute decline in functional status ${ }^{12}$, to be one of the most common reasons for admission to the hospital in patients with advanced cancer and to herald an even greater decline in performance $^{13}$. In fact, the effect on the functional status is prominent. The impairment in the level of functioning may last up to 12 months ${ }^{9-}$ ${ }^{11}$, thus representing a challenge in the process of recovery for the elderly and demented. Further, an association between the length of persistence of delirium symptoms and functional recovery has been documented. The persistence of delirium symptoms for less than two weeks was associated with excellent functional recovery, whereas persistence for a longer time was associated with less favorable outcomes ${ }^{14}$. Even more, delirium predicted greater mortality and accelerated cognitive decline in dementia ${ }^{15-17}$.
The current literature suggests that delirium has an impact on the functional status. Most studies focused on the long-term effect on the functional status, whereas results documenting the acute impact of delirium remain scarce. In order to further explore the impact of delirium on functioning, an analysis in respect to the level of functioning, contributing etiological factors and delirium resolution was performed.

\section{Methods}

\section{Patients}

All patients were recruited from patients referred for delirium management to the Memorial Sloan Kettering Cancer Center (MSKCC) Psychiatry Service from July to November 2000 and from July 2004 to June 2006. MSKCC is a 452 bed private hospital specializing in the treatment of cancer, averaging more than 20,000 admissions every year. The Consultation-Liaison Psychiatry Service performs on average more than 2,000 consultations yearly.

The main inclusion criterion was meeting the DSM-IV-TR ${ }^{18}$ criteria for delirium. Exclusion criteria included patient or family objections to pharmacologic intervention, inability to participate with delirium rating, and severe agitation interfering with the interview.

All patients and their families provided verbal consent to be evaluated and receive antipsychotics for symptomatic relief of delirium. In patients with limited capacity to provide consent, the patient's primary caregiver provided verbal consent alongside the patient's assent to intervention. When patients or families were not willing to be evaluated or did not agree to management with antipsychotics, their data was not recorded. All 
data was obtained from the routine daily clinical care of patients diagnosed with delirium documenting the course of delirium, entered into the IRB-approved-database for subsequent analysis and was a waiver obtained for the analysis.

\section{Measurements}

Delirium severity was measured with the $\mathrm{MDAS}^{20}$, a 10-item, four-point clinician-rated scale (possible range $0-30)^{20}$. Scale items assess the disturbance in arousal and level of consciousness, as well as several areas of cognitive functioning, psychomotor activity, and sleep-wake cycle. An MDAS score of $>10$ identifies the presence of delirium ${ }^{21,22}$. Delirium severity is categorized by using the MDAS scores as follows: mild delirium (MDAS<16), moderate delirium (MDAS 1622 ) and severe delirium (MDAS $>22$ ). The MDAS uses psychomotor behavior for the subtypes of delirium and was based on the predominant presentation, hypoactive or hyperactive psychomotor behavior.

The level of functioning was assessed with the Karnofsky scale of Performance Status (KPS), indicating physical performance ability $^{19}$. The KPS has been validated for the functional assessment of cancer patients and ranges from 10-100. In particular, scores of less than 50 indicate an inability to care for oneself and therefore require hospital care. A score of 40 indicates disability with the need for assistance and a score of 30 indicates severe disability and indicates the need for hospital admission. A score of 20 indicates the necessity of hospital admission in a very sick patient requiring active treatment and a score of 10 indicates being moribund.

MDAS and KPS rating were performed by the fellows of the consultation-liaison psychiatry service who were trained by William Bre- itbart ${ }^{20}$ who established the MDAS. Inter-rater reliability was performed between fellows, however, not formally evaluated as the purpose of the database was the documentation of clinical routine and the course of delirium.

\section{Procedures}

Sociodemographic and medical variables, such as age, sex, cancer diagnosis, stage of cancer (localized, metastatic, or terminal), psychiatric diagnosis including pre-existing dementia, presence of brain metastases, and delirium etiologies were collected at the baseline assessment. Antipsychotic medication was initiated upon diagnosis of delirium and adjusted as determined by clinical response. In accordance with the guidelines for the treatment of delirium ${ }^{23}$, the standard approach was to manage delirium with antipsychotics and continue the necessary medical treatment, including risk factors for delirium such as the administration of opiates or corticosteroids, and to treat reversible underlying causes such as hypoxia and infection.

MDAS, KPS and side effect rating were performed at baseline (T1), repeated at 2-3 days (T2) and 4-7 days (T3), Rating was performed once on the indicated days, usually in the early afternoon. After seven days, the observation period ended. Patients were continued on antipsychotic medication as necessary.

\section{Statistical Analysis}

Data analyses were performed with the Statistical Package for the Social Sciences (SPSS) 20 for Windows. The primary interests were resolved and persistent delirium. The secondary interests were functional recovery versus functional impairment, in addition to sociodemographic and medical variables, as well as delirium severity. 
In a first step, the dataset was defined as a dichotomous variable with two levels: Resolved delirium and persistent delirium. The dichotomous set was analyzed in respect to the level of functioning (KPS). In a second step, medical variables that were associated with an improvement in the level of functioning of less than 10 on the KPS representing patients with functional impairment were identified and then defined as a dichotomous variable accordingly. The comparison group was defined as patients with KPS improvement of more than 10 representing functional recovery. In the functionally-impaired, KPS scores were 22.7 (10-30, SD 5.4) at baseline and, 22.1 (10-30 SD 5.8) at T3. In the functionally-recovered, KPS scores were 25.1 (2040, SD 5.9) and 41.3 (30-70, SD 10.6), respectively. At T3, the level of functioning was superior in the functionally-recovered ( $\mathrm{t}$-test: $\mathrm{t}=-11.4(109), \mathrm{p}<0.001)$.

The t-test for independent variables was computed for continuous variables, such as the age of patients, medication dosing, MDAS and KPS scores between subsets and the oneway ANOVA for multiple, independent measures such as the description of the level of functioning in mild, moderate and severe delirium. The ANOVA repeated measures test was used for repeated measures of dependent variables, such as subsequent MDAS and KPS scores. Pearson's Chi-square test was used for categorical variables such as the prevalence of terminal illness in haloperidolmanaged patients. In order to analyze the effect of medical variables on functional impairment and recovery, multivariate logistic regression analyses were performed.

The significance level alpha $(\alpha)$ was set at $\mathrm{p}<0.05$.

\section{Results}

\section{Baseline characteristics}

Overall, 111 cases of delirium managed with haloperidol, risperidone, olanzapine and aripiprazole for delirium were retrieved. The patients had an advanced age, were predominantly male, and hypoactive delirium and hyperactive delirium were equally distributed. Cancer diagnoses were diverse and delirium was generally multifactorial (table 1).

\section{Description of the level of functioning}

The functional status was impaired at baseline and improved throughout the management period. KPS scores increased from 24.1 at baseline, to 28.6 at $\mathrm{T} 2$ and to 33.0 at $\mathrm{T} 3$ (ANOVA: $\mathrm{F}=80.5, \mathrm{p}<0.001$ ). Surprisingly, delirium severity as measured by mild, moderate and severe delirium did not influence the level of functioning (ANOVA: $\mathrm{F}=1.60, \mathrm{p}=$ $0.207)$. At baseline, KPS scores in mild delirium were 25.5 (10-40, SD 7.2), in moderate delirium 23.2 (20-40, SD 5.1) and severe delirium 24.2 (20-30, SD 5.1). Instead, there were differences in respect to the resolution of delirium: In patients with resolved delirium, KPS scores increased from 24.4 (20-40, SD $5.7)$ at baseline to $30.2(20-60, \mathrm{SD} 10.9)$ at $\mathrm{T} 2$ and 35.1 (10-70, SD 13.2) at T3 (ANOVA: F $=82.2, \mathrm{p}<0.001)$. In patients with persistent delirium, an improvement in KPS scores was noted, but to a lesser amount. KPS scores increased from 23.1 (10-30, SD 6.2) at baseline to 23.5 (10-30, SD 6.9) at T2 and 26.2 (10-40, SD 9.4) at T3 (ANOVA: $F=6.5, p=0.018$ ). Between patients with resolved and persistent delirium, KPS scores were not different at baseline, however, at T2 and T3, KPS scores and thus the level of functioning were higher 
Table 1

Sociodemographic and medical characteristics of patients with functional impairment and functional recovery

\begin{tabular}{|c|c|c|c|}
\hline & $\begin{array}{l}\text { All patients } \\
(\mathrm{N}=111)\end{array}$ & $\begin{array}{l}\text { Functional impairment } \\
\qquad(\mathrm{N}=48)\end{array}$ & $\begin{array}{l}\text { Functional recovery } \\
\qquad(\mathrm{N}=63)\end{array}$ \\
\hline Age & $65.6(23-89$, SD 13.7) & $66.4(29-89$, SD 13.0) & 64.9 (23-85, SD 14.2) \\
\hline \multicolumn{4}{|l|}{ Gender in \% } \\
\hline Male & 41.4 & 58.3 & 58.7 \\
\hline Female & 58.6 & 41.7 & 41.3 \\
\hline Dementia in $\%$ & 19.8 & 22.9 & 17.5 \\
\hline \multicolumn{4}{|l|}{ Diagnoses in \% } \\
\hline Lung & 21.6 & 22.9 & 20.6 \\
\hline Brain & 10.8 & 16.7 & 6.3 \\
\hline Gastrointestinal & 23.4 & 22.9 & 23.8 \\
\hline Genitourinary & 9 & 8.3 & 9.5 \\
\hline Sarcoma & 7.2 & 4.2 & 9.5 \\
\hline Head and neck & 6.3 & 6.3 & 6.3 \\
\hline Gynecological & 9 & 6.3 & 11.1 \\
\hline Endocrine & 2.7 & - & 4.8 \\
\hline Lymphoma & 0.9 & 2.1 & - \\
\hline Skin & 2.7 & 2.1 & 3.2 \\
\hline Hematological & 1.8 & 2.1 & 1.6 \\
\hline Other & 3.6 & 2.1 & 3.2 \\
\hline \multicolumn{4}{|l|}{ Stage in $\%$} \\
\hline Localized & 34.9 & 26.1 & 41.3 \\
\hline Advanced & 47.7 & 45.7 & 49.2 \\
\hline Terminal & 17.4 & 28.3 & 9.5 \\
\hline Brain metastases in \% & 9 & 7.9 & 10.4 \\
\hline \multicolumn{4}{|l|}{ Etiologies in $\%$} \\
\hline Opioids & 88.3 & 81.2 & 93.7 \\
\hline Corticosteroids & 53.2 & 54.2 & 41.3 \\
\hline Infection & 24.3 & 31.2 & 19 \\
\hline Hypoxia & 36.9 & 50 & 27 \\
\hline CNS disease & 12.6 & 27.1 & 12.7 \\
\hline Dehydration & 5.4 & 4.2 & 6.3 \\
\hline Other medication & 81.1 & 87.5 & 82.5 \\
\hline Other & 84.7 & 83.3 & 79.4 \\
\hline Total number of etiologies & $5(3-8$, SD 1$)$ & $5.3(3-8$, SD 1.9) & $4.7(3-7$, SD 0.9$)$ \\
\hline
\end{tabular}

SD (Standard Deviation). 
in patients with resolved delirium indicating functional recovery. At T2, KPS scores were 23.5 (10-30, SD 6.9) in persistent delirium and 30.2 (20-60, SD 10.9) in resolved delir- ium (t-test: $\mathrm{t}=-3(109), \mathrm{p}=0.004)$ and at $\mathrm{T} 3$, KPS scores were 26.2 (10-40, SD 9.4) and $35.1(10-70$, SD 13.2) (t-test: $\mathrm{t}=-3.2(109), \mathrm{p}$ $=0.002)$, respectively.

\section{Table 2}

Multivariate logistic regression analyses of medical variables

\begin{tabular}{|c|c|c|c|c|}
\hline & $\mathrm{B}(\mathrm{SE})$ & Wald's $\chi^{2}$ & Significance & Odds Ratio \\
\hline Lung & $-4.53(1.11)$ & 0.17 & 0.684 & - \\
\hline Brain & $-2.62(1.33)$ & 3.86 & 0.050 & 0.07 \\
\hline Gastrointestinal & $-0.51(1.12)$ & 0.21 & 0.648 & - \\
\hline Genitourinary & $-0.28(1.28)$ & 0.05 & 0.827 & - \\
\hline Sarcoma & $-0.46(1.40)$ & 0 & 0.973 & - \\
\hline Head and neck & $-1.57(1.40)$ & 1.26 & 0.261 & - \\
\hline Gynecological & $0.29(1.25)$ & 0.05 & 0.820 & - \\
\hline Skin & $-0.87(1.68)$ & 0.27 & 0.604 & - \\
\hline Hematological & $-0.24(2.01)$ & 0.01 & 0.788 & - \\
\hline Stage of illness & $-1.26(0.41)$ & 9.54 & 0.002 & 0.28 \\
\hline Opioids & $1.31(0.72)$ & 3.37 & 0.066 & - \\
\hline Corticosteroids & $-0.59(0.48)$ & 1.50 & 0.221 & - \\
\hline Infection & $-0.86(0.53)$ & 2.70 & 0.100 & - \\
\hline Hypoxia & $-1.03(0.48)$ & 4.65 & 0.031 & 0.36 \\
\hline CNS disease & $-0.81(0.79)$ & 1.05 & 0.305 & - \\
\hline Dehydration & $0.65(1.18)$ & 0.30 & 0.584 & - \\
\hline Other medication & $-0.38(0.66)$ & 0.33 & 0.567 & - \\
\hline Other & $-0.45(0.63)$ & 0.50 & 0.480 & - \\
\hline Delirium subtype & $1.01(0.66)$ & 2.37 & 0.124 & - \\
\hline \multicolumn{5}{|c|}{ Delirium resolution at } \\
\hline $\mathrm{T} 2$ & $1.60(0.78)$ & 4.17 & 0.041 & 4.94 \\
\hline T3 & $1.94(0.89)$ & 4.76 & 0.029 & 6.95 \\
\hline
\end{tabular}

$\chi^{2}$ (Chi-Square), SE (Standard Error).

\section{Patients with functional impairment}

Patients with functional recovery or impairment were not different in age, by pre-existing dementia, or brain metastases (table 1 and 3). There was a trend towards brain cancer $(16.7 \%$ and $6.3 \%)$ contributing to functional impairment and terminal cancer contributed to functional impairment $(28.3 \%$ and $9.5 \%)$. Among etiologies, only hypoxia (50\% 
and $27 \%$ ) and multiple etiologies caused more severe functional impairment (table 2).

There were also few differences in management characteristics (table 3). Hypoactive and hyperactive delirium was similarly distributed with $50 \%$ in the functionally-impaired compared to $41.3 \%$ and $58.7 \%$ in the functionally-recovered. There were no differences in respect to the dosing of risperidone, olanzapine or aripiprazole between the functionally-impaired and -recovered. Only, when haloperidol was administered higher doses were given to the functionally-recovered $(2.9 \mathrm{mg}$ and $6.2 \mathrm{mg}$ ). However, there was also a trend towards the functionallyimpaired managed with haloperidol being older (68.3, 42-86, SD 13.3 vs. 58.4, 23-78,
SD 13.9, t-test: $\mathrm{t}=1.82(2), \mathrm{p}=0.078)$ and suffering more often from terminal illness (31.3\% and 5.3\%, $\chi^{2}$ (1) 4.13, p = 0.073).

Furthermore, there were no differences in MDAS scores at baseline (18.2 and 18.3), at T2 and T3 however, delirium was more severe in the functionally-impaired. MDAS scores at T2 were 13.1 and 8.9 and at T3 were 10.6 and 6.0 indicating the functionallyimpaired did not achieve delirium resolution. Although MDAS scores decreased in all patients, the delirium resolution was inferior in the functionally-recovered: At T2, delirium resolution was $63.5 \%$ and $25 \%$ and at $\mathrm{T} 3$, $87.3 \%$ and $62.5 \%$, respectively. On the contrary, functional recovery was associated with shorter delirium.

Table 3

Delirium and management characteristics of patients with functional impairment and functional recovery

$\begin{array}{ccc}\begin{array}{c}\text { Functional impairment } \\ (\mathrm{N}=48)\end{array} & \text { Functional recovery } & \text { Statistics }\end{array}$

$(\mathrm{N}=48) \quad(\mathrm{N}=63)$

\begin{tabular}{|c|c|c|c|}
\hline Age & 66.4 (29-89, SD 13) & $64.9(23-85$, SD 14.2) & $0.56(109), p=0.579^{a}$ \\
\hline Total number of etiologies & $5.3(3-8$, SD 1.9) & $4.7(3-7$, SD 0.9) & $3.05(109), p=0.003^{a}$ \\
\hline \multicolumn{4}{|l|}{ MDAS } \\
\hline Baseline & $18.2(11-30$, SD 5) & $18.3,11-29$, SD 4.3 & $-0.08(109), p=0.935^{\mathrm{a}}$ \\
\hline $\mathrm{T} 2$ & $13.1(4-25$, SD 4.3$)$ & $8.9(1-24$, SD 4.7$)$ & $4.54(109), \mathrm{p}<0.001^{\mathrm{a}}$ \\
\hline $\mathrm{T} 3$ & 10.6 (3-26, SD 5.6) & $6.0(1-17, \mathrm{SD} 3.7)$ & $5.18(109), \mathrm{p}<0.001^{\mathrm{a}}$ \\
\hline Statistics & $\mathrm{F}=95.2, \mathrm{p}<0.001^{\mathrm{b}}$ & $\mathrm{F}=331.2, \mathrm{p}<0.001^{\mathrm{b}}$ & \\
\hline \multicolumn{4}{|l|}{ KPS } \\
\hline Baseline & $22.7(10-30$, SD 5.4) & 25.1 (20-40, SD 5.9) & $-2.18(109), p=0.032^{a}$ \\
\hline $\mathrm{T} 2$ & $22.3(10-30$, SD 5.6) & 33.4 (20-60, SD 33.5) & $-6.55(109), \mathrm{p}<0.001^{\mathrm{a}}$ \\
\hline $\mathrm{T} 3$ & $22.1(10-30$, SD 5.8) & 41.3 (30-70, SD 10.5) & $-11.35(109), \mathrm{p}<0.001^{\mathrm{a}}$ \\
\hline Statistics & $\mathrm{F}=1.8, \mathrm{p}=0.182^{\mathrm{b}}$ & $\mathrm{F}=263.5, \mathrm{p}<0.001^{\mathrm{b}}$ & \\
\hline \multicolumn{4}{|l|}{ Medication at $\mathrm{T} 3$ in $\mathrm{mg}$} \\
\hline Haloperidol & $2.9(1-6$, SD 1.6) & $6.2(1.5-16$, SD 3.6) & $-3.37(33), p=0.002^{a}$ \\
\hline Risperidone & $1.3(0.25-3$, SD 0.8$)$ & $1.3(0.5-3, \mathrm{SD} 0.7)$ & $-.20(30), \mathrm{p}=0.841^{\mathrm{a}}$ \\
\hline Olanzapine & 6.5.(2.5-15, SD 4.5) & $5(2.5-10, \mathrm{SD} 2.2)$ & $5.39(20), p=0.363^{a}$ \\
\hline Aripiprazole & $22.5(15-30$, SD 6.1$)$ & $16.7(10-30$, SD 6.2$)$ & $1.96(19), \mathrm{p}=0.065^{\mathrm{a}}$ \\
\hline
\end{tabular}

Memorial Delirium Assessment Scale (MDAS), Karnofsky Performance Status scale (KPS), SD (Standard Deviation), ${ }^{\mathrm{a}} \mathrm{t}$-test, ${ }^{\mathrm{b}}$ ANOVA repeated measures test. 
Whereas the level of functioning expectedly improved in the functionally-recovered, the functionally-impaired did not achieve improvement in KPS scores or the level of functioning.

\section{Discussion}

These findings suggest that delirium had an acute impact on the level of functioning in the course of hospitalization and that active management of delirium with antipsychotics reversed this effect. On the contrary, shorter delirium was associated with functional recovery. In this respect, these results confirmed previous findings which have shown that delirium has an acute impact on the level of functioning ${ }^{13}$, however, add new insights into the necessity of active management with antipsychotics in order to reverse this effect. Furthermore, brain cancer, terminal illness, hypoxia, and multiple etiologies were confounders for persistent delirium and functional impairment.

It is known that delirium has a long-term effect on cognitive and functional status in older patients with or without dementia ${ }^{9-11}$. Also, an interaction between the length of persistence of delirium symptoms and functional recovery has been documented: The shorter the persistence of delirium symptoms, the better the functional recovery has been. In particular, persistence of delirium for less than two weeks has been associated with excellent functional recovery ${ }^{14}$.

Similarly, within one week of observation, the functional recovery was also superior with a shorter duration of delirium as documented by the functionally-recovered in contrast to the functionally-impaired. Although at baseline, no differences in delirium sever- ity between the functionally-impaired and recovered existed, in the course of management, more severe and persistent delirium became apparent in the functionally-impaired at T2 and T3. Thus, the necessity of actively managing delirium in order to accelerate the functional recovery process and prevent increased morbidity, mortality and rates of institutionalization ${ }^{1}$ ought to be a primary goal and while previously shown in the long-term setting, extended to the acute care setting.

Surprisingly, the severity of delirium, mild, moderate, or severe, did not affect the functional status independently, interesting aspects remain. Persistent delirium had an impact on the functional status in the acute care setting and, in contrast, the level of functioning improved with delirium resolution. This indicated that successful management of delirium with antipsychotics caused the reversal of functional impairment. This effect extended to patients with persistent delirium in whom the level of functioning also improved, however, the improvement was less pronounced.

At baseline, there were no differences in functional status between the functionallyimpaired and -recovered. Over the course of management, the functional status of patients with resolved delirium improved and was superior to patients with persistent delirium. Of interest, this effect appeared to affect all patients with delirium in contrast to previous studies that found persistent delirium in older patients over several months. Still, patients with persistent delirium and lower KPS scores were older than patients with resolved delirium (71.3 and 63.8 years), which implied that advanced age could be associated with a protracted and refractory course of delirium and a more severe and prolonged impact on the level of functioning.

As medical factors may contribute to functional impairment, these variables were identified by examining cases with functional im- 
pairment. There were no differences with respect to age, pre-existing dementia, or brain metastases, except for an increased prevalence of brain cancer, terminal illness, hypoxia, and multiple etiologies in the functionally-impaired. These factors were confounded persistent delirium as has been previously shown $8,24,25$.

With respect to the adequacy of management, the dosing of antipsychotic medication did not appear to have an effect on functional improvement. When risperidone, olanzapine, or aripiprazole were administered, no differences existed between the functionally-impaired and -recovered. Only in patients managed with haloperidol higher doses were administered to the functionally-recovered. However, these patients tended to be older and terminal illness was present at a higher rate, which in part could have explained differences in haloperidol dosing. Overall, the medication doses administered were comparable to those in existing studies ${ }^{26}$.

There are a number of limitations to be noted. Although the design of the data collection was prospective, it was not possible to follow patients prior to the onset of delirium and measure the impact of delirium in a prospective manner. Instead, the reversal of impairment in the level of functioning was described and deduced on the impact of delirium on the level of functioning. Due to the design of the data collection, measuring the first MDAS upon presentation with delirium, it was not possible to obtain a baseline cognitive assessment of functional assessment. Further, the observation period was limited to one week and a longer observation period would have been preferable. In addition, there was a selection of patients with delirium through the referral process, as well as an exclusion of patients unable or unwilling to comply with rating. Although all rater were trained by the creator of the MDAS and inter- rater reliability was performed, no formal evaluation of the inter-rater reliability was recorded. The delirium database contained data of the management of delirium with aripiprazole, haloperidol, risperidone, and haloperidol. Delirium was classified as hypoactive and hyperactive by predominant presentation, the mixed subtype was not assigned. All patients were naturally assigned to a medication, the assignment was not random. All patients had cancer diagnoses and the generalizability of results to the non-cancer population remains to be evaluated. The use of antipsychotics in the management of delirium has not been approved by the regulatory agencies, and the use of antipsychotics in the elderly patients with dementia carries a black box warning of increased risk of death $^{27,28}$. Yet, this analysis may be another step towards demonstrating the acute impact of delirium on the level of functioning, the reversibility of this effect by management with antipsychotics, and warrants future studies with a prospective design.

In summary, this analysis suggests that delirium had an acute impact on the level of functioning which was reversible with the appropriate management of delirium. On the contrary shorter delirium was associated with functional recovery. Brain cancer, terminal illness and hypoxia were confounders to persistent delirium and functional impairment.

\section{References}

1. Inouye SK. Delirium in hospitalized older patients. Clin Geriatr Med 1998;14(4):745-764.

2. Francis J, Martin D, Kapoor WN. A prospective study of delirium in hospitalized elderly. JAMA 1990; 263(8): 1097-1101.

3. Voyer P, McCusker J, Cole MG, St Jacques S, Khomenko L. Factors associated with delirium severity among older patients. J Clin Nurs 2007; 16(5): 819-831. 
4. Bucht G, Gustafson Y, Sandberg O. Epidemiology of Delirium. Dement Geriatr Cogn Disord 1999; 10(5): 315-318.

5. Lipowski ZJ. Delirium in the elderly patient. N Engl J Med 1989; 320(9): 578-582.

6. Bond SM, Neelon VJ, Belyea MJ. Delirium in hospitalized older patients with cancer. Oncol Nurs Forum 2006; 33(6): 1075-1083.

7. Breitbart W, Strout D. Delirium in the terminally ill. Clin Geriatr Med 2000; 16(2): 357-372.

8. Massie MJ, Holland J, Glass E. Delirium in terminally ill cancer patients. Am J Psychiatry 1983; 140(8): 1048-1050.

9. McCusker J, Cole M, Dendukuri N, Belzile E, Primeau F. Delirium in older medical inpatients and subsequent cognitive and functional status: a prospective study. CMAJ 2001; 165(5): 575-583.

10. McCusker J, Cole M, Dendukuri N, Han L, Belzile E. The course of delirium in older medical inpatients: a prospective study. J Gen Intern Med 2003; 18(9): 696-704.

11. McCusker J, Cole MG, Dendukuri N, Belzile E. Does delirium increase hospital stay? J Am Geriatr Soc 2003; 51(11): 1539-1546.

12. Murray AM, Levkoff SE, Wetle TT, Beckett L, Cleary $\mathrm{PD}$, Schor JD, et al. Acute delirium and functional decline in the hospitalized elderly patient. J Gerontol 1993; 48(5): M181-M186.

13. Centeno C, Sanz A, Bruera E. Delirium in advanced cancer patients. Palliat Med 2004; 18(3): 184-194.

14. Kiely DK, Jones RN, Bergmann MA, Murphy KM, Orav EJ, Marcantonio ER. Association between delirium resolution and functional recovery among newly admitted postacute facility patients. J Gerontol A Biol Sci Med Sci 2006; 61(2): 204-208.

15. Kiely DK, Marcantonio ER, Inouye SK, Shaffer ML, Bergmann MA, Yang FM, et al. Persistent delirium predicts greater mortality. J Am Geriatr Soc 2009; 57(1): 55-61.

16. Gross AL, Jones RN, Habtemariam DA, Fong TG, Tommet D, Quach L, et al. Delirium and long-term cognitive trajectory among persons with dementia. Arch Intern Med 2012; 172(17): 1324-1331.

17. Weiner MF. Impact of Delirium on the course of Alzheimer Disease. Arch Neurol 2012; 69(12): 1639-1640.

18. American Psychiatric Association. Diagnostic and Statistical Manual of Mental Disorders, 4th Edition, Text Revision. Washington, DC: American Psychiatric Association; 2000.

19. Karnofsky DA, Burchenal JH. The clinical evaluation of chemotherapeutic agents in cancer. In: Macleod CM, ed- itor. Evaluation of Chemotherapeutic Agents. New York: Columbia University Press; 1949. pp. 191-205.

20. Breitbart W, Rosenfeld B, Roth A, Smith MJ, Cohen K, Passik S. The Memorial Delirium Assessment Scale. J Pain Symptom Manage 1997; 13(3): 128-137.

21. Kazmierski J, Kowman M, Banach M, Fendler W, Okonski P, Banys A, et al. Clinical utility and use of DSMIV and ICD-10 vriteria and The Memorial Delirium Assessment Scale in establishing a diagnosis of delirium after cardiac surgery. Psychosomatics 2008; 49(1): 73-76.

22. Lawlor PG, Nekolaichuk C, Gagnon B, Mancini IL, Pereira JL, Bruera ED. Clinical utility, factor analysis, and further validation of the Memorial Delirium Assessment Scale in patients with advanced cancer: Assessing delirium in advanced cancer. Cancer 2000; 88(12): 2859-2867.

23. Trzepacz PT, Breitbart W, Franklin J, Levenson J, Martini R, Wang P. Practice guideline for the treatment of patients with delirium. American Psychiatric Association. Am J Psychiatry 1999; 156(5 Suppl): 1-20.

24. Breitbart W, Tremblay A, Gibson C. An open trial of olanzapine for the treatment of delirium in hospitalized cancer patients. Psychosomatics 2002; 43(3): 175-182.

25. Lawlor PG, Gagnon B, Mancini IL, Pereira JL, Hanson J, Suarez-Almazor ME, et al. Occurrence, causes, and outcome of delirium in patients with advanced cancer: a prospective study. Arch Intern Med 2000; 160(6): 786-794.

26. Seitz DP, Gill SS, van Zyl LT. Antipsychotics in the treatment of delirium: a systematic review. J Clin Psychiatry 2007; 68(1): 11-21.

27. Jeste DV, Blazer D, Casey D, Meeks T, Salzman C, Schneider L, et al. ACNP White Paper: Update on use of antipsychotic drugs in elderly persons with dementia. Neuropsychopharmacology 2008; 33(5): 957-970.

28. Schneider LS, Dagerman KS, Insel P. Risk of death with atypical antipsychotic drug treatment for dementia: meta-analysis of randomized placebo-controlled trials. JAMA 2005; 19; 294(15): 1934-1943.

Corresponding author:

Soenke Boettger

University Hospital Zürich

Department of Psychiatry and Psychotherapy

Rämistrasse 100, 8091 Zürich

Switzerland

Tel: +41-44-2559700

Fax: +41-44-2559701

E-mail: soenke.boettger@usz.ch 